\title{
Dietary acid load and risk of type 2 diabetes: the E3N-EPIC cohort study
}

\author{
Guy Fagherazzi • Alice Vilier • Fabrice Bonnet • Martin Lajous • Beverley Balkau • \\ Marie-Christine Boutron-Ruault • Françoise Clavel-Chapelon
}

Received: 9 July 2013 / Accepted: 14 October 2013 /Published online: 14 November 2013

(C) Springer-Verlag Berlin Heidelberg 2013

\begin{abstract}
Aims/hypothesis The objective of this study was to evaluate the prospective relationship between dietary acid load, assessed with both the potential renal acid load (PRAL) and the net endogenous acid production (NEAP) scores, and type 2 diabetes risk.

Methods A total of 66,485 women from the E3N-EPIC cohort were followed for incident diabetes over 14 years. PRAL and NEAP scores were derived from nutrient intakes. HRs for type 2 diabetes risk across quartiles of the baseline PRAL and NEAP scores were estimated with multivariate Cox regression models.

Results During follow-up, 1,372 cases of incident type 2 diabetes were validated. In the overall population, the highest PRAL quartile, reflecting a greater acid-forming potential, was associated with a significant increase in type 2 diabetes risk, compared with the first quartile (HR 1.56, 95\% CI 1.29,
\end{abstract}

Electronic supplementary material The online version of this article (doi:10.1007/s00125-013-3100-0) contains peer-reviewed but unedited supplementary material, which is available to authorised users.

G. Fagherazzi · A. Vilier • B. Balkau • M.-C. Boutron-Ruault •

F. Clavel-Chapelon $(\triangle)$

Center for Research in Epidemiology and Population Health (CESP), Inserm U1018, Team 9, Nutrition, Hormones and Women's Health,

Gustave Roussy Institute, 114 rue Edouard Vaillant,

94805 Villejuif Cedex, France

e-mail: francoise.clavel@gustaveroussy.fr

G. Fagherazzi $\cdot$ A. Vilier $\cdot$ B. Balkau $\cdot$ M.-C. Boutron-Ruault $•$

F. Clavel-Chapelon

Paris-South University, Villejuif, France

F. Bonnet

CHU, Rennes, France

M. Lajous

Center for Research on Population Health, National Institute of

Public Health of Mexico, Cuernavaca, Mexico
1.90). The association was stronger among women with BMI $<25 \mathrm{~kg} / \mathrm{m}^{2}$ (HR 1.96, 95\% CI 1.43, 2.69) than in overweight women (HR 1.28, 95\% CI 1.00, 1.64); statistically significant trends in risk across quartiles were observed in both groups ( $p_{\text {trend }}<0.0001$ and $p_{\text {trend }}=0.03$, respectively). The NEAP score provided similar findings.

Conclusions/interpretation We have demonstrated for the first time in a large prospective study that dietary acid load was positively associated with type 2 diabetes risk, independently of other known risk factors for diabetes. Our results need to be validated in other populations, and may lead to promotion of diets with a low acid load for the prevention of diabetes. Further research is required on the underlying mechanisms.

Keywords Acidity $\cdot$ Cohort $\cdot$ Diabetes $\cdot$ Diet $\cdot$ Risk factor

\section{Abbreviations \\ E3N Etude Epidémiologique auprès des femmes de la Mutuelle Générale de l'Education Nationale \\ EPIC European Prospective Investigation into Cancer and Nutrition \\ MET Metabolic equivalent of task \\ NEAP Net endogenous acid production \\ PRAL Potential renal acid load}

\section{Introduction}

A Western diet rich in animal products and other acidogenic foods can induce an acid load that is not compensated for by fruit and vegetables; this can cause chronic metabolic acidosis $[1,2]$. It has recently been suggested that acid/base imbalance may play an important role in some cardiometabolic abnormalities [3-5]. Markers of diet-induced metabolic acidosis 
have been associated with insulin resistance, but only in crosssectional studies [6-8], preventing any conclusions in terms of causality. Moreover, type 2 diabetes incidence and glucose intolerance have been shown to be higher in people with a lower urinary $\mathrm{pH}$ than in those with a higher urinary $\mathrm{pH}$ [9]. Other studies that investigated the effect of an acid/base imbalance on health focused mainly on bone health, kidney stones $[9,10]$ and hypertension; a positive association between hypertension and acid/base imbalance was reported in the Nurses' Health Study II [11], while a more recent prospective study in older Dutch adults from the Rotterdam Study failed to find any association [3].

Two scores are commonly used to estimate dietary acid load in epidemiological studies. The potential renal acid load (PRAL) score takes into account the intestinal absorption rates of contributing nutrient ionic balances for protein, potassium, calcium and magnesium and the dissociation of phosphate at pH $7.4[12,13]$. A negative PRAL value reflects a base (or alkaline)-forming potential, whereas a positive value reflects an acid-forming potential [3]. Frassetto et al [2] developed the net endogenous acid production (NEAP) score, based on total protein and potassium intake as the main components involved in acid production; the NEAP score ranged from 34 to $76 \mathrm{mEq} /$ day in a US population-based report [11]. A Western diet has been characterised by a NEAP score of $\sim 50 \mathrm{mEq} / \mathrm{day}$, although there is large variation in the general population (ranging from 10 to $150 \mathrm{mEq} /$ day) $[2,14,15]$.

To date, no large prospective cohort study has focused on the relationship between dietary acid load and the risk of cardiometabolic diseases. We evaluate prospectively the relation between dietary acid load, using both PRAL and NEAP scores, with the risk of type 2 diabetes in the large E3N-EPIC cohort study.

\section{Methods}

Study cohort The E3N study (Etude Epidémiologique auprès des femmes de la Mutuelle Générale de l'Education Nationale) is a French prospective cohort study of 98,995 female teachers, initiated in 1990. E3N is the French component of the European Prospective Investigation into Cancer and Nutrition (EPIC). Participants returned mailed questionnaires to update health-related information and newly diagnosed diseases every $2-3$ years; in addition, a drug reimbursement claim database has been available since 2004, using medical insurance records (Mutuelle Générale de l'Education Nationale (MGEN)). Average follow-up response rate per questionnaire cycle is $83 \%$, and, overall, the total loss to follow-up since 1990 is below 3\%. All women signed an informed consent letter to comply with the French National Commission for Computerized Data and Individual Freedom (CNIL).
Of the 98,995 women in the cohort, we excluded those who did not complete the dietary questionnaire $(n=24,466)$, those who did not complete any questionnaire after inclusion $(n=1,815)$, prevalent cases of diabetes $(n=830)$, those missing dates of diagnosis of diabetes $(n=58)$, cases with cancer or cardiovascular diseases $(n=4,011)$, and those with extreme (lowest and highest percentile) values for the ratio between energy intake and required energy $(n=1,330)$. A total of 66,485 women were included in the present analysis.

Assessment of dietary information Usual diet over the previous year was assessed by a validated 208-item diet-history questionnaire in 1993, structured according to the French meal pattern. Questions were asked about all times of day when food or drink was consumed, from breakfast to after-dinner snacks, thus including all food and drink intake between meals, such as appetisers before lunch or dinner. Nutrients were then estimated using a French food composition table. The validity and reproducibility of the dietary assessment questionnaire have been reported previously [16].

Assessment of dietary acid load scores and dietary patterns PRAL and NEAP scores were derived from estimations of several nutrient intakes [17]:

1. PRAL $(\mathrm{mEq} /$ day $)=(0.49 \times$ protein $[\mathrm{g} /$ day $])+(0.037 \times$ phosphorus $[\mathrm{mg} /$ day $])-(0.021 \times$ potassium $[\mathrm{mg} /$ day $])$ $-(0.026 \times$ magnesium $[\mathrm{mg} /$ day $])-(0.013 \times$ calcium $[\mathrm{mg} /$ day $])$

2. $\operatorname{NEAP}(\mathrm{mEq} / \mathrm{day})=(54.5 \times$ protein $[\mathrm{g} / \mathrm{day}] /$ potassium $[\mathrm{mEq} /$ day $])-10.2$

Quartiles of the scores were used for statistical analysis.

Ascertainment of diabetes The algorithm used to validate diabetes cases used two steps. A first set of potential cases of diabetes included women who had self-reported diabetes, medical or dietary treatment for diabetes, or hospitalisation for diabetes in at least one of the eight questionnaires returned between 1993 and 2005. A total of 4,289 self-reported cases were identified (see electronic supplementary material [ESM] Fig. 1). Of these, 2,315 cases were considered valid because they were reimbursed for a diabetes drug at least once between 1 January 2004 (date when the file became available) and 30 June 2007 (date of endpoint in the present study). Of the 1,974 remaining cases, women alive and with an accurate address $(n=1,735)$ were mailed a questionnaire that included questions on diagnosis and treatment of diabetes. Among the 1,480 women who completed this questionnaire (response rate $84 \%$ ), we confirmed 342 cases of elevated glucose concentration at diagnosis (fasting $\geq 7.0 \mathrm{mmol} / \mathrm{l}$ or random glucose $\geq 11.1 \mathrm{mmol} / \mathrm{l}$ ), treatment with diabetes drugs, and/or fasting glucose or $\mathrm{HbA}_{1 \mathrm{c}} \geq 7 \%$, $(53.0 \mathrm{mmol} / \mathrm{mol})$, respectively. A total of 2,657 self-reported diabetes cases were thus validated. 
A second strategy to identify diabetes cases used first information exclusively from the drug reimbursement database ( $n=1,216$ participants, who did not self-report any information on diabetes, and had at least one diabetes drug reimbursed). We mailed the diabetes-specific questionnaire to 1,139 of these women (alive with a valid address), and 734 completed it (response rate 64\%). Cases were validated if the patient declared having diabetes and had been reimbursed for diabetes medication at least twice. Women who declared they did not have diabetes and who had been reimbursed for diabetes drugs only once before 30 June $2007(n=233)$ were considered non-cases. We validated as diabetic cases women who confirmed diabetes in the diabetes-specific questionnaire $(n=458)$ and those who did not answer the diabetes-specific questionnaire but had been reimbursed for diabetic drugs at least twice $(n=381)$. Other potential cases were considered non-validated $(n=144)$.

Altogether, a total of 3,496 diabetes cases diagnosed before 30 June 2007 were thus validated cases with incident diabetes in the E3N-EPIC cohort. Although this procedure did not always allow differentiation between type 1 and type 2 diabetes mellitus, given the age range of our population, incident diabetes cases would almost all have been type 2 diabetes. Prevalent cases of diabetes were excluded from our study population, leaving 1,372 validated cases during the follow-up.

Statistical analysis The PRAL and NEAP scores were categorised in quartiles, with the first category as the reference group. Cox multivariate regression models, with age as the time scale, were used to estimate HRs and 95\% CIs. Time at entry was the age at the beginning of follow-up, and exit time was the age when participants were diagnosed with diabetes, died (dates of death were obtained from the participants' medical insurance records), were lost to follow-up or censored at the end of the follow-up period, whichever came first. Multivariate models were adjusted for age, years of education (continuous), and, at baseline, smoking status (non-smoker/ ex-smoker/current smoker), physical activity (continuous, metabolic equivalent of task [MET]-h/week), hypertension (self-reported or use of blood pressure-lowering drugs: yes, no), hypercholesterolaemia (self-reported blood cholesterol $>6.2 \mathrm{mmol} / \mathrm{l}$ or use of cholesterol-lowering drugs: yes, no), family history of diabetes (yes/no), alcohol intake (continuous, g/day), omega 3 fatty acid intake (continuous, g/day), carbohydrate intake (continuous, g/day), energy from fat and protein (continuous, $\mathrm{kJ} /$ day), coffee (continuous, $\mathrm{ml} /$ day), dietary patterns (Western or healthy; for further information, please see Cottet et al [18]) and the following established dietary risk factors for diabetes in the $\mathrm{E} 3 \mathrm{~N}$ cohort: sugar and artificially sweetened beverages (continuous, $\mathrm{ml} /$ day), fruit and vegetables (continuous, g/day), processed meat consumption (continuous, g/day). Since adiposity has been found to be a key factor in the relationship between diet and type 2 diabetes and at least partly an intermediate factor, we tested multivariate models with and without BMI (continuous, $\mathrm{kg} / \mathrm{m}^{2}$ ). When data were missing for fewer than $5 \%$ of adjustment variables, we replaced missing values with the median value.

Tests for linear trend across quartiles of PRAL and NEAP scores were performed using the median value in each quartile.

A cubic spline regression model was also computed to evaluate the shape of the relationship between the PRAL score and the risk of type 2 diabetes. The reference value for estimating HRs and 95\% CIs was chosen as PRAL= $-50 \mathrm{mEq} / \mathrm{day}$, and three knots were used $(\mathrm{PRAL}=-14,-3$ and $7 \mathrm{mEq} / \mathrm{day}$, the quartiles of this score).

All statistical analyses used SAS V.9.3 (SAS Institute, Cary, NC, USA; PHREG procedure for Cox models). All statistical tests were two-sided and considered to be statistically significant if $p<0.05$.

Additional analyses We stratified analyses by BMI categories ( $<25 \mathrm{vs} \geq 25 \mathrm{~kg} / \mathrm{m}^{2}$ ) to test if associations were similar in normal-weight and overweight women. To test a reverse causation hypothesis, we also evaluated the associations between PRAL and NEAP scores with type 2 diabetes by excluding cases that occurred during the first 3 and the first 7 years of follow-up, respectively.

\section{Results}

Dietary acid load and baseline characteristics The correlation between the PRAL and NEAP scores was high $(\rho=0.93)$. Therefore, we only described baseline characteristics of the study population according to the PRAL score. As shown in Table 1, women with a higher dietary acid load (higher PRAL score) were younger than women with a low dietary acid load. They were also characterised by less physical activity, fewer were treated for hypercholesterolaemia, they more often had a family history of diabetes, and they showed better adherence to a Western dietary pattern and, inversely, worse adherence to a healthy dietary pattern. Means (or frequencies) for these variables all differed significantly from each other across the PRAL quartiles - all corresponding $p$ values were highly significant. In contrast, mean BMI (although the corresponding $p$ value was significant) and the proportions of smokers were very similar across quartiles of the PRAL score.

Dietary acid load and nutrient intake A diet with high acid load was associated with higher daily energy intake compared with that of women with low PRAL scores. High-PRAL diets were characterised by a lower percentage of carbohydrates, a higher percentage of fat and a higher percentage of protein 
Table 1 Characteristics of the women studied by quartiles of the PRAL score (E3N-EPIC cohort data, $N=66,485$ )

\begin{tabular}{|c|c|c|c|c|c|}
\hline \multirow[t]{2}{*}{ Characteristic } & \multicolumn{4}{|c|}{ PRAL score quartiles (mEq/day) ${ }^{\mathrm{a}}$} & \multirow[t]{2}{*}{$p$ value $^{\mathrm{b}}$} \\
\hline & $<-14(n=16,622)$ & -14 to $-3(n=16,621)$ & -3 to $7(n=16,621)$ & $\geq 7(n=16,621)$ & \\
\hline PRAL (mEq/day) ${ }^{\mathrm{c}}$ & -23.0 & -8.0 & 1.7 & 14.3 & $<0.0001$ \\
\hline NEAP (mEq/day) & $31.5 \pm 5.4^{\mathrm{d}}$ & $40.7 \pm 3.1$ & $47.5 \pm 3.2$ & $58.2 \pm 7.2$ & $<0.0001$ \\
\hline \multicolumn{6}{|l|}{ Basic } \\
\hline Age (years) & $53.9 \pm 6.8$ & $53.2 \pm 6.7$ & $52.3 \pm 6.5$ & $51.4 \pm 6.2$ & $<0.0001$ \\
\hline BMI $\left(\mathrm{kg} / \mathrm{m}^{2}\right)$ & $22.8 \pm 3.1$ & $22.8 \pm 3.0$ & $22.8 \pm 3.1$ & $22.9 \pm 3.4$ & $<0.0001$ \\
\hline Overweight or obese (\%) & 19.0 & 18.1 & 18.3 & 19.9 & $<0.0001$ \\
\hline Educational level, beyond secondary (\%) & 84.4 & 86.3 & 87.3 & 86.9 & $<0.0001$ \\
\hline Current smoker (\%) & 13.9 & 13.4 & 13.4 & 13.1 & 0.4327 \\
\hline Alcohol abstainers (\%) & 14.1 & 11.3 & 10.9 & 12.4 & $<0.0001$ \\
\hline Intake among alcohol users (g/day) & $13.3 \pm 14.9$ & $13.1 \pm 14.0$ & $13.0 \pm 13.5$ & $13.3 \pm 13.7$ & 0.1379 \\
\hline Physical activity (MET-h/week) & $58.2 \pm 31.6$ & $55.9 \pm 30.5$ & $53.6 \pm 29.5$ & $51.6 \pm 28.7$ & $<0.0001$ \\
\hline Hypertension (\%) & 13.6 & 13.3 & 12.9 & 12.9 & 0.1944 \\
\hline Treated hypercholesterolaemia (\%) & 5.7 & 5.4 & 4.7 & 3.4 & $<0.0001$ \\
\hline Family history of diabetes (\%) & 8.7 & 9.1 & 9.1 & 10.2 & $<0.0001$ \\
\hline \multicolumn{6}{|l|}{ Nutrient intake } \\
\hline Energy (kJ/day) & $8,887.3 \pm 2,296.8$ & $8,796.9 \pm 2,169.2$ & $9,120.5 \pm 2,205.6$ & $10,257.6 \pm 2,400.7$ & $<0.0001$ \\
\hline Carbohydrates ( $\%$ of energy) & $45.2 \pm 7.0$ & $43.6 \pm 6.7$ & $42.6 \pm 6.7$ & $40.8 \pm 7.2$ & $<0.0001$ \\
\hline Fat $(\%$ of energy) & $35.0 \pm 5.4$ & $36.0 \pm 5.2$ & $36.8 \pm 5.2$ & $38.0 \pm 5.6$ & $<0.0001$ \\
\hline Protein (\% of energy) & $17.3 \pm 2.8$ & $17.7 \pm 2.5$ & $18.2 \pm 2.5$ & $19.0 \pm 2.6$ & $<0.0001$ \\
\hline Animal protein (g/day) & $57.7 \pm 18.9$ & $61.3 \pm 17.2$ & $66.6 \pm 17.2$ & $80.6 \pm 20.7$ & $<0.0001$ \\
\hline Plant protein (g/day) & $32.1 \pm 10.4$ & $29.5 \pm 9.5$ & $29.9 \pm 9.6$ & $32.6 \pm 10.7$ & $<0.0001$ \\
\hline Fibre (g/day) & $28.9 \pm 8.6$ & $24.4 \pm 7.1$ & $23.2 \pm 6.9$ & $23.4 \pm 7.1$ & $<0.0001$ \\
\hline Phosphorus (mg/day) & $1,426.2 \pm 395.6$ & $1,387.3 \pm 354.0$ & $1,440.5 \pm 352.1$ & $1,658.2 \pm 399.9$ & $<0.0001$ \\
\hline Potassium (mg/day) & $4,594.5 \pm 1,199.6$ & $3,788.9 \pm 855.7$ & $3,564.4 \pm 838.5$ & $3,550.6 \pm 844.4$ & $<0.0001$ \\
\hline Calcium (mg/day) & $1,058.7 \pm 367.7$ & $1,007.6 \pm 336.7$ & $1,026.1 \pm 336.2$ & $1,172.0 \pm 400.4$ & $<0.0001$ \\
\hline Magnesium (mg/day) & $518.1 \pm 164.0$ & $437.0 \pm 127.0$ & $406.6 \pm 121.0$ & $402.1 \pm 118.6$ & $<0.0001$ \\
\hline Sodium (mg/day) & $2,611.7 \pm 838.3$ & $2,649.2 \pm 810.3$ & $2,801.2 \pm 829.0$ & $3,300.3 \pm 973.5$ & $<0.0001$ \\
\hline \multicolumn{6}{|l|}{ Food intake } \\
\hline Meat (g/day) & $97.6 \pm 48.7$ & $110.4 \pm 47.1$ & $124.9 \pm 48.1$ & $154.5 \pm 58.7$ & $<0.0001$ \\
\hline Fish (g/day) & $32.7 \pm 24.4$ & $33.0 \pm 22.8$ & $33.6 \pm 23.2$ & $36.3 \pm 25.0$ & $<0.0001$ \\
\hline Milk products (g/day) & $237.5 \pm 185.8$ & $222.5 \pm 169.5$ & $213.7 \pm 160.9$ & $209.4 \pm 164.7$ & $<0.0001$ \\
\hline Cheese (g/day) & $41.6 \pm 32.0$ & $46.4 \pm 32.5$ & $54.2 \pm 35.0$ & $78.3 \pm 50.2$ & $<0.0001$ \\
\hline Fruit (g/day) & $82.4 \pm 124.1$ & $67.2 \pm 92.6$ & $55.9 \pm 81.7$ & $45.6 \pm 71.7$ & $<0.0001$ \\
\hline Vegetables (g/day) & $361.2 \pm 159.6$ & $310.6 \pm 133.2$ & $284.9 \pm 121.9$ & $270.0 \pm 121.0$ & $<0.0001$ \\
\hline Potatoes (g/day) & $62.6 \pm 56.9$ & $61.5 \pm 48.1$ & $60.9 \pm 45.0$ & $63.6 \pm 43.8$ & 0.0026 \\
\hline Bread (g/day) & $108.8 \pm 69.6$ & $117.9 \pm 70.1$ & $130.7 \pm 73.9$ & $158.2 \pm 89.1$ & $<0.0001$ \\
\hline Coffee (ml/day) & $395.9 \pm 344.6$ & $313.6 \pm 247.3$ & $254.4 \pm 217.2$ & $202.7 \pm 197.7$ & $<0.0001$ \\
\hline Sugar-sweetened beverages $(\mathrm{ml} / \text { day })^{\mathrm{e}}$ & $47.6 \pm 70.3$ & $43.7 \pm 67.1$ & $47.1 \pm 72.3$ & $49.0 \pm 68.4$ & 0.0362 \\
\hline Artificially sweetened beverages $(\mathrm{ml} / \mathrm{day})^{\mathrm{e}}$ & $78.7 \pm 124.6$ & $76.9 \pm 114.7$ & $75.1 \pm 115.5$ & $94.3 \pm 157.1$ & 0.0446 \\
\hline Western pattern score ${ }^{f}$ & $-0.4 \pm 0.9$ & $-0.2 \pm 0.9$ & $0.04 \pm 0.9$ & $0.5 \pm 1.0$ & $<0.0001$ \\
\hline Healthy pattern score ${ }^{\mathrm{f}}$ & $0.3 \pm 1.0$ & $-0.01 \pm 0.9$ & $-0.2 \pm 0.9$ & $-0.2 \pm 1.0$ & $<0.0001$ \\
\hline
\end{tabular}

${ }^{a}$ As characteristics are very similar according to the NEAP score, baseline characteristics are only presented according to the PRAL score quartiles

${ }^{\mathrm{b}} \chi^{2}$ tests for qualitative variables and ANOVA tests for quantitative variables

${ }^{\mathrm{c}}$ Values are medians per quartile

${ }^{\mathrm{d}}$ Mean $\pm \mathrm{SD}$ (all such values)

${ }^{\mathrm{e}}$ Among consumers only

${ }^{\mathrm{f}}$ For further information on dietary patterns, please see Cottet et al [18] 
(mainly driven by a higher animal protein intake) compared with low-PRAL diets. A high-PRAL score was also characterised by higher intakes of phosphorus, calcium and sodium and a lower magnesium intake.

Dietary acid load and food consumption With respect to specific food groups, a high-PRAL diet included significantly more meat, fish, cheese, bread and soft drinks, particularly artificially sweetened beverages, whereas a diet with a lowPRAL score included more dairy products, fruit, vegetables and coffee (Table 1).

Dietary acid load and risk of type 2 diabetes During the 14 years of follow-up, a total of 1,372 validated cases of incident type 2 diabetes were identified. Both the PRAL and NEAP scores were associated with an increased risk of incident type 2 diabetes in age-adjusted and multivariate models (Table 2). In multivariate model M1, there was a statistically significant linear trend of increasing type 2 diabetes risk with increasing score $\left(p_{\text {trend }}<0.0001\right)$ for both PRAL and NEAP scores. Women with a high acid load (PRAL $>7 \mathrm{mEq} /$ day) were at a higher risk of type 2 diabetes than those with high alkaline load (PRAL $<-14$ mEq/day) (HR 1.71, 95\% CI 1.40, 2.07). An HR of similar magnitude was observed for the NEAP score between extreme categories (HR 1.74, 95\% CI 1.44, 2.11). Adjustment for BMI (multivariate model M2,
Table 2) slightly attenuated the associations between PRAL (or NEAP) and type 2 diabetes risk (HR 1.56, 95\% CI 1.29, 1.90 for PRAL $>7 \mathrm{mEq} /$ day vs $\mathrm{PRAL}<-14 \mathrm{mEq} /$ day; $\left.p_{\text {trend }}<0.0001\right)$. We also tested an adjustment for BMI as a continuous variable and additionally including a squared term in BMI, but it did not modify the associations (data not shown).

Spline regression model We observed a threshold effect in the relationship between PRAL score treated as a continuous variable and the risk of type 2 diabetes (Fig. 1). There was a plateau for HRs associated with negative PRAL values, while risk linearly increased with increasing PRAL when PRAL values became positive. The shape of the relationship was very similar for the NEAP score (not shown).

Stratification by BMI To evaluate whether BMI could modulate the association between dietary acid load and type 2 diabetes risk, we stratified the analyses by BMI $(<$ and $\geq 25 \mathrm{~kg} / \mathrm{m}^{2}$ ) (Table 3). PRAL and NEAP scores were associated with higher type 2 diabetes risk in both normal-weight and overweight/obese women, but the associations were stronger in normal-weight vs overweight women: HRs associated with high-PRAL diet (PRAL $>7 \mathrm{mEq} /$ day) vs low-PRAL diet (PRAL $<-14 \mathrm{mEq} /$ day) were 1.96 (95\% CI 1.43, 2.69) in normal-weight women and 1.28 (95\% CI 1.00, 1.64) in overweight women. Findings were similar for NEAP scores.

Table 2 Age-adjusted and multivariable-adjusted HR for the risk of incident type 2 diabetes according to quartile groups of the PRAL and NEAP scores in the overall population (E3N-EPIC cohort data, $N=66,485$ )

\begin{tabular}{|c|c|c|c|c|}
\hline \multirow[t]{2}{*}{ Dietary acid load score } & \multirow[t]{2}{*}{ Cases } & \multicolumn{3}{|l|}{ HR $(95 \%$ CI) } \\
\hline & & Age-adjusted model & Multivariate model $\mathrm{M} 1^{\mathrm{a}}$ & Multivariate model $\mathrm{M} 2^{\mathrm{b}}$ \\
\hline \multicolumn{5}{|l|}{ PRAL (mEq/day) } \\
\hline$<-14.0$ & 290 & 1 & 1 & 1 \\
\hline-14.0 to -3.0 & 308 & $1.10(0.94,1.29)$ & $1.15(0.98,1.37)$ & $1.17(0.99,1.38)$ \\
\hline-3.0 to 7.0 & 302 & $1.12(0.95,1.32)$ & $1.12(0.93,1.34)$ & $1.16(0.97,1.39)$ \\
\hline$\geq 7.0$ & 472 & $1.87(1.61,2.16)$ & $1.71(1.40,2.07)$ & $1.56(1.29,1.90)$ \\
\hline$p_{\text {trend }}$ & & $<0.0001$ & $<0.0001$ & $<0.0001$ \\
\hline \multicolumn{5}{|l|}{ NEAP (mEq/day) } \\
\hline$<37.2$ & 275 & 1 & 1 & 1 \\
\hline 37.2 to 44.0 & 307 & $1.18(1.00,1.39)$ & $1.11(0.94,1.32)$ & $1.13(0.95,1.33)$ \\
\hline 44.0 to 51.3 & 326 & $1.30(1.10,1.53)$ & $1.16(0.97,1.39)$ & $1.19(1.00,1.42)$ \\
\hline$\geq 51.3$ & 464 & $1.96(1.69,2.28)$ & $1.74(1.44,2.11)$ & $1.57(1.30,1.89)$ \\
\hline$p_{\text {trend }}$ & & $<0.0001$ & $<0.0001$ & $<0.0001$ \\
\hline
\end{tabular}

${ }^{a}$ Models were age-adjusted models, and multivariate models M1 were further adjusted for years of education (continuous), smoking status (non-smoker/ ex-smoker/current smoker), physical activity (continuous, MET-h/week), hypertension (self-reported or use of antihypertensive drugs: yes, no), hypercholesterolaemia (self-reported blood cholesterol $>6.2 \mathrm{mmol} / 1$ or use of cholesterol-lowering drugs: yes, no), family history of diabetes (yes/ no), alcohol intake (continuous, g/day), omega 3 fatty acid intake (continuous, g/day), carbohydrate intake (continuous, g/day), total energy intake (excluding alcohol and carbohydrate, continuous, $\mathrm{kJ} /$ day), coffee (continuous, $\mathrm{ml} / \mathrm{day}$ ), sugar and artificially sweetened beverages (ml/day), fruits and vegetables and processed meat consumption (continuous, $\mathrm{g} /$ day) and dietary patterns (Western or healthy)

${ }^{\mathrm{b}}$ Models M2 were multivariate models $\mathrm{M}_{1}$ further adjusted for BMI (continuous, $\mathrm{kg} / \mathrm{m}^{2}$ ) 


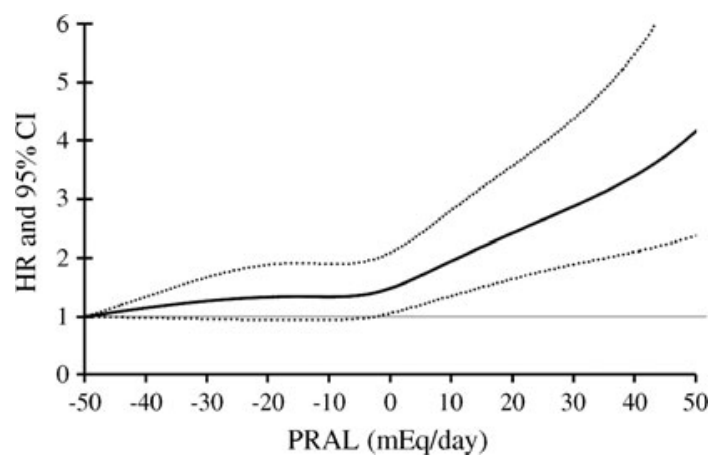

Fig. 1 Cubic spline regression model between PRAL score and risk of type 2 diabetes (E3N-EPIC cohort data, $N=66,485$ ). Spline regression (three knots, reference value: $\mathrm{PRAL}=-50 \mathrm{mEq} / \mathrm{day}$ ). Continuous line, HR. Dashed lines, 95\% CIs. The model was adjusted for age, years of education (continuous), smoking status (non-smoker/ex smoker/current smoker), physical activity (continuous, MET-h/week), hypertension (selfreported or use of antihypertensive drugs: yes, no), hypercholesterolaemia (self-reported blood cholesterol $>6.2 \mathrm{mmol} / \mathrm{l}$ or use of cholesterollowering drugs: yes, no), family history of diabetes (yes/no), alcohol intake (continuous, g/day), omega 3 fatty acid intake (continuous, g/day), carbohydrate intake (continuous, g/day), total energy intake (excluding alcohol and carbohydrate, continuous, $\mathrm{kJ} / \mathrm{day}$ ), consumption of coffee (continuous, ml/day), sugar and artificially sweetened beverages ( $\mathrm{ml} /$ day), fruit, vegetables and processed meat (continuous, g/day) and dietary patterns (Western or healthy)

Reverse causation In order to test a reverse causation hypothesis between PRAL and NEAP scores and type 2 diabetes risk, we excluded the 188 cases that occurred during the first 3 years of follow-up, leaving 1,184 cases for analysis. For both scores, associations with type 2 diabetes were identical with those of the main analyses in terms of magnitude and statistical significance (not tabulated). For example, women with a PRAL $>7 \mathrm{mEq} /$ day were at a similarly increased type 2 diabetes risk compared with women with PRAL $<-14 \mathrm{mEq} /$ day (HR 1.72, 95\% CI 1.39, 2.13, $p_{\text {trend }}<0.001$ ), as in the main analyses. Similar results were obtained when the first 7 years of follow-up were excluded.

\section{Discussion}

In this study of women with relatively alkaline diets (median PRAL $=-3.0 \mathrm{mEq} /$ day), a diet with a high acid load (characterised by a high PRAL or a high NEAP score) was associated with an increased risk of type 2 diabetes. Associations tended to be stronger in normal-weight women than in women with excess weight (BMI $\geq 25 \mathrm{~kg} / \mathrm{m}^{2}$ ).

To our knowledge, this is the first prospective study to evaluate the risk of type 2 diabetes associated with scores reflecting the acid load of the diet, such as PRAL or NEAP. In the Nurses' Health Study II, a high NEAP score was found to be associated with a higher risk of hypertension [11], while this was not confirmed in a recent study using both the PRAL and NEAP scores [3]. In the latter report, based on the Rotterdam Study with 2,241 participants, the multivariate HR comparing the third with the first PRAL tertile was 1.02 .

Table 3 HR for the risk of incident type 2 diabetes according to quartiles of the PRAL and NEAP scores, stratified by BMI (E3N-EPIC Cohort data, $N=66,485)$

\begin{tabular}{|c|c|c|c|c|}
\hline \multirow[t]{2}{*}{ Dietary acid load score ${ }^{a}$} & \multicolumn{2}{|c|}{$\mathrm{BMI}<25 \mathrm{~kg} / \mathrm{m}^{2}$} & \multicolumn{2}{|c|}{$\mathrm{BMI} \geq 25 \mathrm{~kg} / \mathrm{m}^{2}$} \\
\hline & Cases & $\operatorname{HR}(95 \% \mathrm{CI})$ & Cases & $\operatorname{HR}(95 \% \mathrm{CI})$ \\
\hline \multicolumn{5}{|l|}{ PRAL (mEq/day) } \\
\hline$<-14.0$ & 108 & 1 (reference) & 182 & 1 (reference) \\
\hline-14.0 to -3.0 & 125 & $1.19(0.91,1.55)$ & 183 & $1.09(0.88,1.35)$ \\
\hline-3.0 to 7.0 & 111 & $1.07(0.80,1.44)$ & 191 & $1.09(0.87,1.37)$ \\
\hline$\geq 7.0$ & 185 & $1.96(1.43,2.69)$ & 287 & $1.28(1.00,1.64)$ \\
\hline$p_{\text {trend }}$ & & $<0.0001$ & & 0.0573 \\
\hline \multicolumn{5}{|l|}{ NEAP (mEq/day) } \\
\hline$<37.2$ & 107 & 1 (reference) & 168 & 1 (reference) \\
\hline 37.2 to 44.0 & 121 & $1.21(0.92,1.58)$ & 186 & $1.01(0.82,1.26)$ \\
\hline 44.0 to 51.3 & 121 & $1.19(0.89,1.58)$ & 205 & $1.06(0.85,1.32)$ \\
\hline$\geq 51.3$ & 180 & $2.04(1.50,2.76)$ & 284 & $1.25(0.98,1.60)$ \\
\hline$p_{\text {trend }}$ & & $<0.0001$ & & 0.0478 \\
\hline
\end{tabular}

${ }^{a}$ Models were adjusted for years of education (continuous), smoking status (non-smoker/ex-smoker/current smoker), physical activity (continuous, MET-h/week), hypertension (self-reported or use of antihypertensive drugs: yes, no), hypercholesterolaemia (self-reported blood cholesterol $>6.2 \mathrm{mmol} / 1$ or use of cholesterol-lowering drugs: yes, no), family history of diabetes (yes/no), alcohol intake (continuous, g/day), omega 3 fatty acid intake (continuous, g/day), carbohydrate intake (continuous, g/day), total energy intake (excluding alcohol and carbohydrate, continuous, kJ/day), coffee (continuous, $\mathrm{ml} /$ day), sugar and artificially sweetened beverages ( $\mathrm{ml} /$ day), fruit, vegetable and processed meat consumption (continuous, $\mathrm{g} / \mathrm{day}$ ), dietary patterns (Western or healthy) and BMI (continuous, $\mathrm{kg} / \mathrm{m}^{2}$ ) 
However, there was little variability in either the PRAL or NEAP scores, which may have limited the possibility to detect associations. Other studies that investigated the effect of an acid/base imbalance on health focused on bone and kidney stones $[9,10]$. Associations between low serum bicarbonate, higher anion gap and low urine $\mathrm{pH}$-all markers of metabolic acidosis - and insulin resistance have been reported [7, 19, 20]. Higher levels of serum lactate, a component of the anion gap, have been associated with a higher risk of type 2 diabetes in the ARIC cohort [20]. A recent prospective study showed that higher plasma bicarbonate levels correlated with a lower odds of incident type 2 diabetes among women in the Nurses' Health Study [21]. Type 2 diabetes incidence and glucose intolerance have been shown to be much higher in people with a lower urinary $\mathrm{pH}$ than in normal volunteers [9].

Mediated by adiposity? Although the associations were slightly attenuated when adjusted for BMI, high PRAL and NEAP scores remained strongly associated with an increased risk of type 2 diabetes. Thus it is unlikely that the association between acid load and type 2 diabetes risk is strongly mediated by adiposity. Moreover, associations were slightly stronger in normal-weight than overweight women. Consequently, our results seem to be largely independent of adiposity.

Biological mechanisms The association between dietary acid load and cardiometabolic diseases has been little studied, and few mechanisms have been proposed to explain the potential harmfulness of an acidogenic diet on these diseases.

A diet rich in animal protein may favour net acid intake, while most fruits and vegetables constitute base precursors. In our study, the fact that the association between both PRAL and NEAP scores and the risk of incident type 2 diabetes persisted after adjustment for dietary patterns, meat consumption and intake of fruit, vegetables, coffee and sweetened beverages suggests that dietary acids may play a specific role in promoting the development of type 2 diabetes, irrespective of the foods or drinks that provide the acidic or alkaline components.

A recent review suggested that imbalances in acid/base homeostasis may play an important role in some cardiometabolic abnormalities [4]: an acidogenic diet may cause chronic metabolic acidosis over time, which may then lead to insulin resistance and the metabolic syndrome. This mechanism was supported by cross-sectional studies showing that a high dietary acid load was associated with insulin resistance [6-8]. Results from our prospective study are consistent with these previous findings.

In addition, the induction of mild metabolic acidosis by the administration of ammonium chloride results, in humans, in reduced insulin sensitivity, as assessed by the gold standard technique, the euglycaemic clamp [22]. Furthermore, metabolic acidosis decreases the binding of insulin to its receptors in rats, suggesting that metabolic acidosis may promote insulin resistance [23]. Finally, in experimental studies, acid/base alterations are associated with decreased insulin secretion [24].

Strengths and limitations This study has some limitations. Information on diet was not updated during follow-up, while dietary habits may have changed over time. Although we adjusted for most of the known and potential type 2 diabetes risk factors, residual confounding cannot be ruled out. Finally, our study population included only women. This limitation should be minor, as few studies report differences in risk factors for diabetes between men and women, and proposed biological mechanisms are similar for both sexes.

This study has numerous strengths. The detailed dietary questionnaire of the E3N-EPIC cohort study enables us to characterise women with an acidogenic diet in terms of major food groups and nutrient intakes. Moreover, we analysed validated incident type 2 diabetes cases only, based on a well-defined validation algorithm, which reduces the risk of missing cases or of false positive cases. Some residual misclassification with respect to diabetes status may exist, but it is likely to be non-differential. Finally, to test a reverse causation hypothesis, the prospective design and the long follow-up in the E3N-EPIC cohort allowed us to perform sensitivity analyses, while keeping sufficient statistical power to detect associations, making the reverse causation hypothesis unlikely.

Public health implications This is the first prospective cohort study to show that a dietary acid load is directly associated with an increased risk of type 2 diabetes. From a public health perspective, dietary recommendations should not only incriminate specific food groups but also include recommendations on the overall quality of the diet, notably the need to maintain an adequate acid/base balance.

Acknowledgements We are indebted to all participants in the study and are grateful to the E3N-EPIC group.

Funding The study is supported by the Mutuelle Générale de l'Education Nationale, the Institut de Cancérologie Gustave Roussy, and the Institut National de la Santé et de la Recherche Médicale. The validation of potential diabetes cases was supported by the European Union (Integrated Project LSHM-CT-2006-037197 in the Framework Programme 6 of the European Community) InterAct project. Study sponsors had no role in the design of the study, the analysis or interpretation of data, the writing of the manuscript, or the decision to submit the manuscript for publication.

Duality of interest The authors declare that there is no duality of interest associated with this manuscript.

Contribution statement GF and BB designed the research; GF and AV conducted the research; AV analysed data; GF, FB, ML, MCBR and BB interpreted the data; GF drafted the article, and AV, BB, ML, FB, MCBR 
and FCC revised it critically; FCC contributed substantially to acquisition of the data and had primary responsibility for the final content of the manuscript; all authors read and approved the final manuscript.

\section{References}

1. Lennon EJ, Lemann J Jr (1968) Influence of diet composition on endogenous fixed acid production. Am J Clin Nutr 21:451-456

2. Frassetto LA, Todd KM, Morris RC Jr, Sebastian A (1998) Estimation of net endogenous noncarbonic acid production in humans from diet potassium and protein contents. Am J Clin Nutr 68:576-583

3. Engberink MF, Bakker SJ, Brink EJ et al (2012) Dietary acid load and risk of hypertension: the Rotterdam Study. Am J Clin Nutr 95: $1438-1444$

4. Adeva MM, Souto G (2011) Diet-induced metabolic acidosis. Clin Nutr 30:416-421

5. van den Berg E, Hospers FA, Navis G et al (2011) Dietary acid load and rapid progression to end-stage renal disease of diabetic nephropathy in Westernized South Asian people. J Nephrol 24:11-17

6. Cameron MA, Maalouf NM, Adams-Huet B, Moe OW, Sakhaee K (2006) Urine composition in type 2 diabetes: predisposition to uric acid nephrolithiasis. J Am Soc Nephrol 17:1422-1428

7. Maalouf NM, Cameron MA, Moe OW, Adams-Huet B, Sakhaee K (2007) Low urine $\mathrm{pH}$ : a novel feature of the metabolic syndrome. Clin J Am Soc Nephrol 2:883-888

8. Souto G, Donapetry C, Calvino J, Adeva MM (2011) Metabolic acidosis-induced insulin resistance and cardiovascular risk. Metab Syndr Relat Disord 9:247-253

9. Sakhaee K, Adams-Huet B, Moe OW, Pak CY (2002) Pathophysiologic basis for normouricosuric uric acid nephrolithiasis. Kidney Int 62:971-979

10. Reddy ST, Wang CY, Sakhaee K, Brinkley L, Pak CY (2002) Effect of low-carbohydrate high-protein diets on acid-base balance, stoneforming propensity, and calcium metabolism. Am J Kidney Dis 40: 265-274
11. Zhang L, Curhan GC, Forman JP (2009) Diet-dependent net acid load and risk of incident hypertension in United States women. Hypertension 54:751-755

12. Remer T, Manz F (1994) Estimation of the renal net acid excretion by adults consuming diets containing variable amounts of protein. Am J Clin Nutr 59:1356-1361

13. Remer T, Manz F (1995) Potential renal acid load of foods and its influence on urine pH. J Am Diet Assoc 95:791-797

14. Frassetto LA, Morris RC Jr, Sebastian A (2006) A practical approach to the balance between acid production and renal acid excretion in humans. J Nephrol 19(Suppl 9):S33-S40

15. Sebastian A, Frassetto LA, Sellmeyer DE, Merriam RL, Morris RC Jr (2002) Estimation of the net acid load of the diet of ancestral preagricultural Homo sapiens and their hominid ancestors. Am J Clin Nutr 76:1308-1316

16. van Liere MJ, Lucas F, Clavel F, Slimani N, Villeminot S (1997) Relative validity and reproducibility of a French dietary history questionnaire. Int J Epidemiol 26(Suppl 1):S128-S136

17. Remer T, Dimitriou T, Manz F (2003) Dietary potential renal acid load and renal net acid excretion in healthy, free-living children and adolescents. Am J Clin Nutr 77:1255-1260

18. Cottet V, Touvier M, Fournier A et al (2009) Postmenopausal breast cancer risk and dietary patterns in the E3N-EPIC prospective cohort study. Am J Epidemiol 170:1257-1267

19. Farwell WR, Taylor EN (2008) Serum bicarbonate, anion gap and insulin resistance in the National Health and Nutrition Examination Survey. Diabet Med 25:798-804

20. Crawford SO, Hoogeveen RC, Brancati FL et al (2010) Association of blood lactate with type 2 diabetes: the Atherosclerosis Risk in Communities Carotid MRI Study. Int J Epidemiol 39:1647-1655

21. Mandel EI, Curhan GC, Hu FB, Taylor EN (2012) Plasma bicarbonate and risk of type 2 diabetes mellitus. CMAJ 184:E719-E725

22. DeFronzo RA, Beckles AD (1979) Glucose intolerance following chronic metabolic acidosis in man. Am J Physiol 236:E328-E334

23. Whittaker J, Cuthbert C, Hammond VA, Alberti KG (1982) The effects of metabolic acidosis in vivo on insulin binding to isolated rat adipocytes. Metabolism 31:553-557

24. Rebolledo OR, Hernandez RE, Zanetta AC, Gagliardino JJ (1978) Insulin secretion during acid-base alterations. Am J Physiol 234: E426-E429 